\title{
Correlation of survivin and B-cell lymphoma 2 expression with pathological malignancy and anti-apoptotic properties of glial cell tumors
}

\author{
IN-SUK BAE, CHOONG-HYUN KIM, JAE-MIN KIM, JIN-HWAN CHEONG, JE-IL RYU and MYUNG-HOON HAN \\ Department of Neurosurgery, Hanyang University Guri Hospital, Guri-si, Gyeonggi-do 11923, Republic of Korea
}

Received December 15, 2016; Accepted January 27, 2017

DOI: $10.3892 / \mathrm{br} .2017 .861$

\begin{abstract}
Apoptosis, whose mechanism remains unclear, is regulated by multiple factors. B-cell lymphoma 2 (Bcl-2) is a well-known anti-apoptotic mediator. Survivin is also a recently recognized novel family inhibitor of apoptosis protein, which inhibits apoptosis via a pathway distinct from Bcl-2 family members. Survivin and Bcl-2 are expressed in various types of human cancer. In the present study, survivin and Bcl-2 expression were characterized in glial cell tumors, and the correlation with pathological malignancy and anti-apoptotic properties were investigated. Fifty-eight patients who had undergone surgical resection for glial cell tumors were evaluated. The pathological types of glial cell tumors were categorized according to the World Health Organization classification. Survivin and Bcl-2 expression levels were investigated by western blot analysis, and apoptosis was detected by DNA fragmentation analysis. The anti-apoptotic rate of glial cell tumors was calculated in tumor samples according to the expression of survivin and Bcl-2 or co-expression. Survivin was characterized in $60.3 \%$, and Bcl-2 was expressed in $43.1 \%$ of glioma samples. Co-expression of survivin and Bcl-2 was observed in $25.9 \%$ of the tumor specimens. Survivin expression in astrocytic tumors was identified to be significantly associated with the pathological grade $(\mathrm{P}<0.05)$; however, Bcl-2 was not $(\mathrm{P}>0.05)$. Anti-apoptotic rate of glial cell tumors were detected in 91.4, 92.0 and $100 \%$ of patients exhibiting survivin, Bcl-2 or co-expression, respectively. However, the difference in anti-apoptotic frequency between the three groups was not identified to be statistically significant $(\mathrm{P}>0.05)$. The present study suggests that survivin expression is correlated with pathological grades of gliomas. In addition, the expression of survivin or Bcl-2 exerts potent anti-apoptotic
\end{abstract}

Correspondence to: Professor Choong-Hyun Kim, Department of Neurosurgery, Hanyang University Guri Hospital, 153 Kyoungchun-ro, Guri-si, Gyeonggi-do 11923, Republic of Korea E-mail: kch5142@hanyang.ac.kr

Key words: glioma, survivin, B-cell lymphoma 2, apoptosis, malignancy properties in gliomas. Thus, survivin or Bcl-2 may serve as potential targets for inducing the apoptosis of gliomas.

\section{Introduction}

Glial cell tumors form the most common group of primary brain tumors, accounting for $40 \%$ of all central nervous system (CNS) neoplasms (1). Despite marked efforts to improve the clinical outcome of glioma patients, very little progress has been made, particularly in patients with high-grade gliomas (2). As in other types of cancer, malignant progression of glial cell tumors is accompanied by abnormal molecular changes (3). Various molecules are vital in the pro- or anti-survival signaling pathways (4). Therefore, the identification and development of chemotherapeutic agents that selectively target molecular events associated to malignant progression may be an effective strategy for the treatment of glial cell tumors (5).

The molecular mechanisms of tumorigenesis and malignant progression of tumor cells have been identified to be correlated with the inactivation of tumor suppressor genes or the overexpression of oncogenes. However, the mechanisms of tumorigenesis and malignant progression of glial cell have remained unclear. Survivin is a bifunctional member of the inhibitor of apoptosis protein (IAP) family that counteracts cell death and controls mitotic progression. The expression of survivin is among the most tumor specific of all human genes. It is undetectable in the majority of healthy adult tissues, but becomes prominently expressed in nearly all types of human cancer. Tumors with overexpression of survivin generally have a worse prognosis and are associated with resistance to radiotherapy or chemotherapy (6).

By contrast, B-cell lymphoma $2(\mathrm{Bcl}-2)$ is one of a family of interacting proteins involved in the regulation of controlled cell death, apoptosis. Bcl-2 appears to inhibit apoptosis by preventing cells from responding to stimuli to initiate apoptosis. Apoptosis is a recognized feature of malignant glioma, however, the association with $\mathrm{Bcl}-2$ expression remains unclear. Furthermore, it is unclear whether Bcl-2 expression is associated with the pathological grade of glial cell tumors. In a previous study, survivin expression was significantly associated with $\mathrm{Bcl}-2$ expression and anti-apoptotic properties, which were strongly correlated with poor clinical outcome following resection in gastric and colorectal cancers (7). 
The aim of the present study was to analyze survivin and $\mathrm{Bcl}-2$ expression in glial cell tumors, and investigate the correlation of survivin and Bcl-2 expression with pathological malignancy and anti-apoptotic properties.

\section{Materials and methods}

Patient population. Tumor specimens from 58 patients with glial cell tumors were prospectively collected from consecutive patients who had undergone curative resection between July 2003 and December 2004 at the Department of Neurosurgery, Hanyang University Guri Hospital (Guri-si, South Korea). Donors provided informed consent. The pathological types were categorized according to the World Health Organization classification (8). Clinical data were reviewed for each medical record and follow-up information was retrieved from medical records, including neuroradiological images. All tumor specimens were immediately frozen following surgical removal and stored in a liquid nitrogen tank (at $-80^{\circ} \mathrm{C}$ ) until performance of experimental procedures. The study protocol was approved by the Institutional Review Board of Hanyang University Medical Center.

Western blot analysis. Tumor specimens were washed three times for 15 min each time in phosphate-buffered saline (PBS) and lysed using a homogenization buffer containing $0.1 \mathrm{M}$ sodium phosphate buffer $(\mathrm{pH} 6.1), 1 \mathrm{mM}$ ethylenediaminetetraacetic acid (EDTA), $1 \mathrm{mM}$ dithiothreitol, $0.1 \mathrm{mM}$ phenylmethylsulfonyl fluoride and $1 \mathrm{mM}$ benzamidine (1\%). Following protein quantification using the DC Protein Assay (Bio-Rad Laboratories, Inc., Hercules, CA, USA), $15 \mu \mathrm{g}$ protein was loaded into each lane and subjected to SDS-PAGE on $10 \%$ acrylamide gel. Proteins were transferred overnight onto polyvinylidene difluoride membranes (Bio-Rad Laboratories, Inc.) at a constant voltage $(40 \mathrm{~V})$. Membranes were saturated for $1 \mathrm{~h}$ using 5\% dry milk in Tris-buffered saline with Tween-20 [10 mM Tris-buffer ( $\mathrm{pH} 7.5), 100 \mathrm{mM} \mathrm{NaCl}$ and $0.1 \%$ Tween-20] and incubated overnight with $1 \mu \mathrm{g} / \mathrm{ml}$ anti-survivin antibody (cat. no. NB500-238; Novus Biologicals LLC, Littleton, CO, USA) or Bcl-2 monoclonal antibody (cat. no. sc-7382; Santa Cruz Biotechnology, Inc., Dallas, TX, USA) at $4^{\circ} \mathrm{C}$. The membranes were then incubated for $1 \mathrm{~h}$ with a mouse anti-mouse immunoglobulin $\mathrm{G}$ antibody conjugated to alkaline phosphatase (cat. no. CN55455; Zymed Laboratories, Inc., San Francisco, CA, USA). Detection was then performed using an ECL detection system (GE Healthcare Life Sciences, Arlington Height, IL, USA). The blots were washed in PBS for $2 \mathrm{~min}$, and the bands were visualized using ECL plus (GE Healthcare Life Sciences) and autoradiography (Fig. 1).

DNA fragmentation analysis. Tumor specimens were washed with PBS ( $\mathrm{pH}$ 7.4) three times and homogenized with lysis buffer (10 mM Tris-HCl, $100 \mathrm{mM}$ EDTA and 0.5\% SDS; $\mathrm{pH}$ 8.0). The homogenized samples were centrifuged at 15,000 $\mathrm{x}$ g for $5 \mathrm{~min}$ at $4^{\circ} \mathrm{C}$ and $50 \mu \mathrm{g}$ of the supernatants were placed in fresh tubes. During the time course experiments, the experimental cells and control cells (normal glial cells) were terminated at the same time. Samples were dissolved in $20 \mu \mathrm{l}$ sample buffer (50 mM Tris-HCl, 0.05\% SDS and 10 mM EDTA; pH 8.0) and incubated with $10 \mathrm{mg} / \mathrm{ml}$ heat-treated RNase A (Sigma-Aldrich
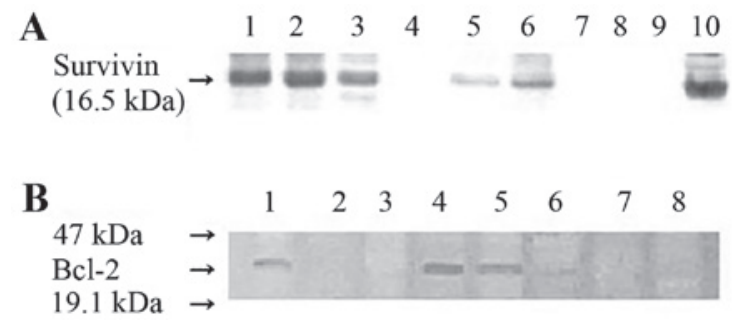

Figure 1. (A) Western blot analysis of survivin protein expression using monoclonal anti-survivin antibody. Positive expression is apparent in lanes 1-3, 5, 6 and 10. (B) Bcl-2 expression analysis by western blotting using Bcl-2 monoclonal antibody. Positive expression was observed in lanes 1, 4 and 5 . Bcl-2, B-cell lymphoma 2.

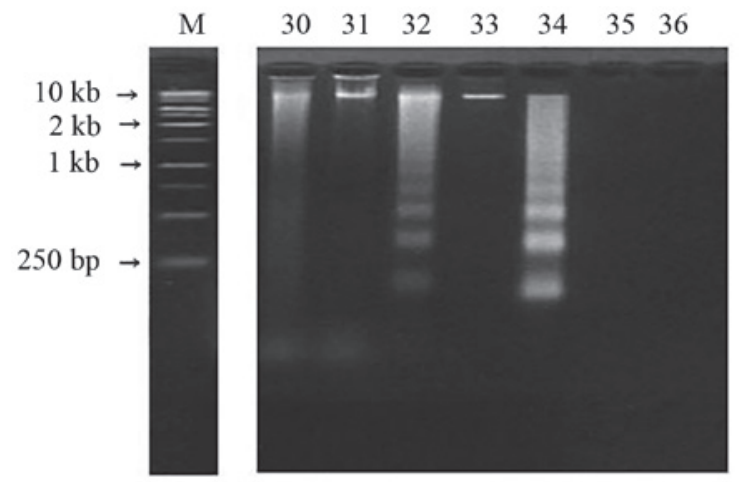

Figure 2. DNA fragmentation in agarose gel. Fragmented DNA was isolated and electrophoresed in a $2.0 \%$ agarose gel containing $50 \mathrm{ng} / \mathrm{ml}$ ethidium bromide. A typical laddering pattern represents apoptosis in cases 32 and 34 . M, marker.

Corporation; Merck KGaA, Darmstadt, Germany) at $56^{\circ} \mathrm{C}$ for 1 h. Proteinase K $(10 \mathrm{mg} / \mathrm{ml}$; Promega Corporation, Madison, WI, USA) was added to each sample and incubated for $1 \mathrm{~h}$ at $37^{\circ} \mathrm{C}$. The reaction was stopped by increasing the temperature to $70^{\circ} \mathrm{C}$ for $10 \mathrm{~min}$. Five microliters of loading buffer $(20 \%$ glycerol, $20 \mathrm{mM}$ EDTA and $0.15 \%$ bromphenol blue) were added and the samples were analyzed on $2 \%$ agarose gels in Tris/boric acid/EDTA buffer ( $89 \mathrm{mM}$ Tris base, $89 \mathrm{mM}$ boric acid and $2.5 \mathrm{mM}$ EDTA; $\mathrm{pH} 8.0$ ) containing $50 \mathrm{ng} / \mathrm{ml}$ ethidium bromide. Electrophoresis was performed at $100 \mathrm{~V}$ for $50 \mathrm{~min}$ and DNA fragmentations were visualized by ultraviolet transillumination (Fig. 2). An anti-apoptotic rate was defined as the percentage of patients that did not exhibit apoptosis.

Statistical analysis. Statistical analysis was performed using SPSS 10 software (SPSS, Inc., Chicago, IL, USA). The difference between survivin and Bcl-2 expression levels according to the pathological type of brain tumor was analyzed using the Kruskal-Wallis test. To determine whether there was an association between anti-apoptosis and survivin or Bcl-2 expression levels, or between the co-expression of survivin and $\mathrm{Bcl}-2$, a $\chi^{2}$ test was performed. $\mathrm{P}<0.05$ was considered to indicate a statistically significant difference.

\section{Results}

Demographic data and tumor pathology. The patient groups comprised of 32 males and 26 females with a median age of 
Table I. Expression levels of survivin and Bcl-2 protein, and apoptosis in 58 patients with glial cell tumors.

\begin{tabular}{|c|c|c|c|}
\hline \multirow[b]{2}{*}{ Pathological type } & \multicolumn{2}{|c|}{ Expression rate, $\mathrm{n}(\%)$} & \multirow[b]{2}{*}{ Apoptosis $\mathrm{n}(\%)$} \\
\hline & Survivin & Bcl-2 & \\
\hline \multicolumn{4}{|l|}{ Astrocytic tumor, $n=45$} \\
\hline Astrocytoma, $n=15$ & $7(46.7)$ & $9(60.0)$ & $4(26.6)$ \\
\hline Anaplastic astrocytoma, $n=7$ & $4(57.1)$ & $1(14.3)$ & $2(28.5)$ \\
\hline Glioblastoma, $\mathrm{n}=23$ & $17(73.3)^{\mathrm{a}}$ & $7(30.4)$ & $7(30.4)$ \\
\hline \multicolumn{4}{|l|}{ Oligodendroglial tumor, $n=6$} \\
\hline Oligodendroglioma, $\mathrm{n}=2$ & $2(100.0)$ & $2(100.0)$ & $0(0.0)$ \\
\hline Anaplastic oligodendroglioma, $\mathrm{n}=4$ & $2(50.0)$ & $3(75.0)$ & $1(25.0)$ \\
\hline \multicolumn{4}{|l|}{ Ependymal tumor, $n=7$} \\
\hline Ependymoma, $n=4$ & $1(25.0)$ & $2(50.0)$ & $0(0.0)$ \\
\hline Anaplastic ependymoma $n=3$ & $2(66.7)$ & $1(33.3)$ & $0(0.0)$ \\
\hline Total, $\mathrm{n}=58$ & $35(60.3)$ & $25(43.1)$ & $14(24.1)$ \\
\hline
\end{tabular}

${ }^{\mathrm{a}} \mathrm{P}<0.05$ glioblastoma vs. astrocytoma and anaplastic astrocytoma (Kruskal-Wallis test). Bcl-2 was not correlated with pathological malignancy. Bcl-2, B-cell lymphoma-2.

Table II. Association between apoptosis, and survivin and Bcl-2 expression levels in 58 patients with glial cell tumors.

\begin{tabular}{lccr}
\hline & \multicolumn{2}{c}{ Apoptosis, $\mathrm{n}(\%)$} & \\
\cline { 2 - 3 } Expression & $(+)$ & $(-)$ & P-value $^{\mathrm{a}}$ \\
\hline $\begin{array}{l}\text { Survivin expression } \\
\text { Positive, } \mathrm{n}=35\end{array}$ & $3(8.6)$ & $32(91.4)$ & \\
$\quad$ Negative, $\mathrm{n}=23$ & $11(47.8)$ & $12(52.2)$ & \\
Bcl-2 expression & & & 0.001 \\
Positive, $\mathrm{n}=25$ & $2(8.0)$ & $23(92.0)$ & \\
$\quad$ Negative, $\mathrm{n}=3$ & $12(36.4)$ & $21(63.6)$ & \\
Survivin and Bcl-2 & & & 0.000 \\
expression & & & \\
Positive, $\mathrm{n}=15$ & $0(0.0)$ & $15(100.0)$ & \\
Negative, $\mathrm{n}=13$ & $9(69.2)$ & $4(30.8)$ & \\
\hline
\end{tabular}

${ }^{\mathrm{a}} \chi^{2}$-test. Bcl-2, B-cell lymphoma 2.

40 years (range, 6-68 years). The tumor specimens consisted of 45 astrocytic tumors (comprising 15 astrocytomas, 7 anaplastic astrocytomas and 23 glioblastomas), 6 oligodendroglial tumors (including 2 oligodendrogliomas and 4 anaplastic oligodendrogliomas) and 7 ependymal tumors (4 ependymomas and 3 anaplastic ependymomas).

Expression levels of survivin and Bcl-2, and apoptosis in tumor specimens. Survivin and Bcl-2 expression levels were analyzed by western blotting in 58 brain tumor specimens. As demonstrated in Fig. 1, survivin and Bcl-2 were detected in the specimens from the majority of tumor types. The positive expression of survivin was exhibited in $35(60.3 \%)$ of the 58 patients. A high expression level of survivin was observed in glioblastomas.
The frequency of survivin expression in the tumor samples was significantly correlated with the pathological grade of glial cell tumors $(\mathrm{P}<0.05$; Table I). Positive $\mathrm{Bcl}-2$ protein expression was observed in $25(43.1 \%)$ of 58 patients, although its expression was not correlated with the pathological grade. Survivin and Bcl-2 were co-expressed in 15 (25.9\%) patients.

Apoptosis in tumor specimens was investigated using DNA fragmentation. A typical laddering pattern of DNA fragmentation is presented in Fig. 2. Apoptosis was confirmed in 14 (24.1\%) of the 58 glial cell tumor samples obtained from patients (Table I).

Association between survivin and Bcl-2 expression levels, and anti-apoptosis. To investigate the anti-apoptotic rate and its association with survivin and/or Bcl-2 expression in glial cell tumor specimens, the anti-apoptotic rate was compared between the survivin-positive, Bcl-2-positive and co-expression groups. Anti-apoptosis effects were detected in 32 (91.4\%) of 35 patients for survivin-positive tumors, while anti-apoptosis effects for Bcl-2-positive tumors were found in 23 (92\%) of 25 patients. An anti-apoptosis effect was observed in all cases of co-expression of survivin- and Bcl-2-positive tumors. A statistically significant association was observed between an anti-apoptosis effect and the expression of survivin or Bcl-2, and the co-expression of survivin and Bcl-2 ( $\mathrm{P}<0.05$; Table II). However, no difference in the frequency of anti-apoptosis was identified between the three groups, including patients expressing survivin, Bcl-2, or patients co-expressing survivin and Bcl-2 ( $\mathrm{P}>0.05)$. The anti-apoptotic rate between the positive and negative expression groups of survivin, Bcl-2 or survivin and Bcl-2 was identified to be significantly different ( $\mathrm{P}=0.003$; Fig. 3$)$.

\section{Discussion}

Survivin is a unique member of the IAP family. It is of special interest in oncology, as it is strongly expressed in 


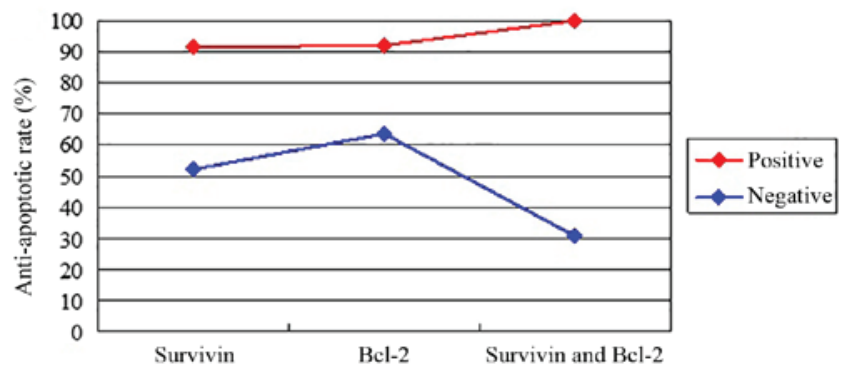

Figure 3. Line graph displaying the anti-apoptotic rate between positive and negative expression groups of survivin, Bcl-2, or survivin and Bcl-2 in 58 samples of glial cell tumor (Kruskal-Wallis test, $\mathrm{P}=0.003$ ). Bcl-2, B-cell lymphoma 2.

embryonic and fetal organs, undetectable in the majority of healthy adult tissues, and markedly overexpressed in various types of human cancer $(9,10)$. In the present study, survivin was expressed in 35 of 58 glial cell tumor patient samples. Furthermore, survivin expression was identified to be correlated with pathological grades of astrocytic tumors and anti-apoptosis in glial cell tumors. In addition, the co-expression of survivin and Bcl-2 exerted a potent anti-apoptotic influence, although, did not exert a synergistic effect on the anti-apoptotic properties of glial cell tumors. The current results indicate that proteins involved in anti-apoptosis are actively expressed in glial cell tumors. Proteins, such as survivin and $\mathrm{Bcl}-2$ may, therefore, promote the malignant progression of glial cell tumors.

Novel anti-apoptotic proteins that signal through caspase-dependent and -independent mechanisms have previously been described. Six members of this IAP family have been introduced in humans: HIAP1, HIAP2, XIAP, NIAP, survivin and livin (11). Survivin is a member of the IAP family that directly inhibits caspase- 3 and -7 activity and regulates the $\mathrm{G} 2 / \mathrm{M}$ phase of the cell cycle. Its gene is encoded at chromosome 17q25. Furthermore, survivin links to microtubules of the mitotic spindle (12). Interruption of survivin-microtubule interactions resulted in the loss of anti-apoptotic properties and increased caspase- 3 activity during mitosis. The over-expression of survivin in cancer may eliminate apoptotic checkpoints and allow aberrant progression of transformed cells through mitosis.

Expression of survivin has been reported in various types of human cancer, including lung, stomach, colon, pancreas, breast and prostate, as well as neuroblastomas, melanomas, and lymphomas $(7,13)$. Survivin is normally expressed during development, but it is undetectable in healthy adult tissues and prominently overexpressed in human cancers. However, the timing of its overexpression during tumorigenesis and malignant progression remains unknown. Survivin was identified to be expressed in primary brain tumors, particularly in glioblastomas, meningiomas and schwannomas $(14,15)$. The present results demonstrate that survivin was expressed at high levels in high-grade glial cell tumors. A previous study indicated that survivin expression in human cancers was associated with a more aggressive and invasive clinical phenotype, and correlated with the inhibition of apoptosis (7). IAPs may have greater anti-apoptotic properties than any other family of apoptotic inhibitors, including Bcl-2.
The expression of survivin has been significantly correlated with the malignant grade of astrocytic tumors and a worse clinical outcome (16). Furthermore, patients who had been diagnosed with glioblastomas, with over-expression of survivin, have been observed to have a significantly shorter survival time (17). Therefore, it is suggested that survivin may be involved in tumorigenesis and malignant progression of glial cell tumors (18). Therefore, survivin may be a useful indicator for predicting the clinical outcome of patients and may be an ideal target for novel chemotherapeutic agents. Consequently, survivin inhibition may manage glial cell tumors without affecting normal brain tissue (19). In the current study, the association between survivin expression and pathological malignancy of astrocytic tumors was also consistent with previous findings (16). However, the association between survivin expression and the pathological malignancy of oligodendrogliomas or ependymomas has not been confirmed.

Various previous studies have shown a correlation between survivin expression and radiation resistance in certain human cancers. Chakravarti et al (17) reported that survivin improves double-strand DNA break repair in tumor cells, inhibiting radiation-induced cell death. Sasaki et al (15) demonstrated that suppression of survivin by small interfering ribonucleic acid improved the radiosensitivity of tumor cells. Based on these results, anti-survivin therapeutic strategies may enhance the radiation response and be correlated with favorable clinical outcomes (20).

Bcl-2 regulates cytochrome $\mathrm{c}$ release from mitochondria and suppresses upstream initiation of the caspase activation cascade. Bcl-2 acts to extend cell survival by blocking apoptosis and may, therefore, influence pathological malignancy (21). In the current study, Bcl-2 expression was not correlated with a malignant grade of astrocytic cell tumors. Numerous authors previously reported that cytoplasmic $\mathrm{Bcl}-2$ expression levels were higher in low-grade astrocytomas than in high-grade astrocytomas and were not associated with the pathological malignancy of astrocytic cell tumors.

Survivin and Bcl-2 possess potent anti-apoptotic properties. Survivin reportedly affects caspase- 3 or -7 via various signaling pathways of the Bcl-2 family. In addition, Bcl-2 expression was identified to be positively correlated with survivin expression in epithelial ovarian cancer (22). However, survivin expression was not correlated with $\mathrm{Bcl}-2$ expression in glial cell tumors (16). Due to these varying results, the anti-apoptotic effect of survivin remains controversial and is not clearly understood. Low survivin expression levels in certain human cancer cells were associated with apoptotic properties and correlated with Bcl-2 expression (23). Conversely, survivin expression in bladder tumors was not associated with high expression levels of Bcl-2. This result was not surprising, due to the fact that survivin and Bcl-2 do not share completely common mechanisms of transcriptional activation (11). However, the expression levels of survivin and Bcl-2 in melanomas may provide two independent mechanisms of apoptosis inhibition leading to malignant progression of cancer cells (24).

Survivin and Bcl-2 expression in breast cancer cells is significantly involved in apoptosis inhibition, which potentially contributes to malignant progression of cancer cells and resistance to chemotherapy or radiotherapy. Survivin 
expression was significantly associated with Bcl-2 expression and anti-apoptotic properties, which were strongly correlated with a worse clinical outcome following resection in gastric and colorectal cancers (7). Expression of the survivin gene alone or the survivin gene plus other anti-apoptotic genes, such as Bcl-2 may have more noticeable anti-apoptotic properties in breast carcinomas (13). However, in the current study, no synergistic anti-apoptotic effects were noted in the co-expression group using glial cell tumor samples. Differences in the results may arise from the small number of glial cell tumors, which may be unrepresentative due to the tumor heterogeneity.

A major limitation to verifying the hypothesis of the current study is the small number of glial cell tumor samples. Additionally, anti-apoptosis in the same pathology of glial cell tumors was not quantitatively evaluated. Thus, studies of other molecular markers, such as other IAP members, and investigations into the response to treatments, such as radiotherapy and chemotherapy, are required. Furthermore, the correlation between molecular markers and the clinical outcome of glial cell tumors requires investigation. Larger prospective studies using the same pathology of glial cell tumors must be performed to determine the association between apoptosis, and survivin and $\mathrm{Bcl}-2$ expression levels.

Previous studies have indicated that survivin is an ideal target for cancer treatment, either directly or by affecting endothelial cell viability (25). A previously reported study on survivin antisense treatment demonstrated that survivin caused regression in vascular capillary formation (26). Thus, survivin antisense treatment has anti-angiogenic effects and anti-tumor cell effects, and may present as an attractive target for glial cell tumor treatment.

In conclusion, the current results indicate that survivin or Bcl-2 expression exert potent anti-apoptotic effects in glial cell tumors. In addition, survivin expression in astrocytic tumors was identified to be significantly associated with pathological malignancy. Survivin or Bcl-2 may be attractive targets for use in cancer therapy to target tumor cells while leaving the normal cells unaffected. Thus, suppression of survivin or Bcl-2 expression may be an effective treatment modality against glial cell tumors.

\section{References}

1. Ahmed R, Oborski MJ, Hwang M,Lieberman FS and Mountz JM: Malignant gliomas: Current perspectives in diagnosis, treatment, and early response assessment using advanced quantitative imaging methods. Cancer Manag Res 6: 149-170, 2014.

2. Corwin D, Holdsworth C, Rockne RC, Trister AD, Mrugala MM, Rockhill JK, Stewart RD, Phillips M and Swanson KR: Toward patient-specific, biologically optimized radiation therapy plans for the treatment of glioblastoma. PLoS One 8: e79115, 2013.

3. Kanamori M, Higa T, Sonoda Y, Murakami S, Dodo M, Kitamura H, Taguchi K, Shibata T, Watanabe M, Suzuki H, et al: Activation of the NRF2 pathway and its impact on the prognosis of anaplastic glioma patients. Neuro-oncol 17: 555-565, 2015.

4. Hu B, Emdad L, Bacolod MD, Kegelman TP, Shen XN, Alzubi MA, Das SK, Sarkar D and Fisher PB: Astrocyte elevated gene-1 interacts with Akt isoform 2 to control glioma growth, survival, and pathogenesis. Cancer Res 74: 7321-7332, 2014.

5. Würth R, Pattarozzi A, Gatti M, Bajetto A, Corsaro A, Parodi A, Sirito R, Massollo M, Marini C, Zona G, et al: Metformin selectively affects human glioblastoma tumor-initiating cell viability: A role for metformin-induced inhibition of Akt. Cell Cycle 12: $145-156,2013$
6. Yamamoto $\mathrm{T}$ and Tanigawa $\mathrm{N}$ : The role of survivin as a new target of diagnosis and treatment in human cancer. Med Electron Microsc 34: 207-212, 2001.

7. Kawasaki H, Altieri DC, Lu CD, Toyoda M, Tenjo T and Tanigawa N: Inhibition of apoptosis by survivin predicts shorter survival rates in colorectal cancer. Cancer Res 58: 5071-5074, 1998.

8. Louis DN, Ohgaki H, Wiestler OD and Cavenee WK: World Health Organization histological classification of tumours of the central nervous system. International Agency for Research on Cancer, Lyon, 2007.

9. Altieri DC: The molecular basis and potential role of survivin in cancer diagnosis and therapy. Trends Mol Med 7: 542-547, 2001.

10. Altieri DC: Validating survivin as a cancer therapeutic target. Nat Rev Cancer 3: 46-54, 2003.

11. Ashhab Y, Alian A, Polliack A, Panet A and Ben Yehuda D: Two splicing variants of a new inhibitor of apoptosis gene with different biological properties and tissue distribution pattern. FEBS Lett 495: 56-60, 2001

12. O'Connor DS, Grossman D, Plescia J, Li F, Zhang H, Villa A, Tognin S, Marchisio PC and Altieri DC: Regulation of apoptosis at cell division by $\mathrm{p} 34 \mathrm{cdc} 2$ phosphorylation of survivin. Proc Natl Acad Sci USA 97: 13103-13107, 2000.

13. Tanaka $\mathrm{K}$, Iwamoto $\mathrm{S}$, Gon $\mathrm{G}$, Nohara $\mathrm{T}$, Iwamoto $\mathrm{M}$ and Tanigawa N: Expression of survivin and its relationship to loss of apoptosis in breast carcinomas. Clin Cancer Res 6: 127-134, 2000.

14. Kleinschmidt-DeMasters BK, Heinz D, McCarthy PJ, Bobak JB, Lillehei KO, Shroyer AL and Shroyer KR: Survivin in glioblastomas. Protein and messenger RNA expression and comparison with telomerase levels. Arch Pathol Lab Med 127: 826-833, 2003.

15. Sasaki T, Lopes MBS, Hankins GR and Helm GA: Expression of survivin, an inhibitor of apoptosis protein, in tumors of the nervous system. Acta Neuropathol 104: 105-109, 2002.

16. Kajiwara Y, Yamasaki F, Hama S, Yahara K, Yoshioka H, Sugiyama K, Arita K and Kurisu K: Expression of survivin in astrocytic tumors: Correlation with malignant grade and prognosis. Cancer 97: 1077-1083, 2003.

17. Chakravarti A, Noll E, Black PM, Finkelstein DF, Finkelstein DM, Dyson NJ and Loeffler JS: Quantitatively determined survivin expression levels are of prognostic value in human gliomas. J Clin Oncol 20: 1063-1068, 2002.

18. Xie D, Zeng YX, Wang HJ, Wen JM, Tao Y, Sham JS and Guan XY: Expression of cytoplasmic and nuclear Survivin in primary and secondary human glioblastoma. Br J Cancer 94: 108-114, 2006.

19. Altieri DC: Survivin, cancer networks and pathway-directed drug discovery. Nat Rev Cancer 8: 61-70, 2008.

20. Kanwar RK, Cheung CH, Chang JY and Kanwar JR: Recent advances in anti-survivin treatments for cancer. Curr Med Chem 17: 1509-1515, 2010.

21. Plati J, Bucur O and Khosravi-Far R: Apoptotic cell signaling in cancer progression and therapy. Integr Biol 3: 279-296, 2011.

22. Zhang SL, Zhao CQ, Lin B, Li Y and Gao H: Expression of survivin gene and its relation with the expression of bcl-2 and bax protein in epithelial ovarian cancer. Zhonghua Fu Chan $\mathrm{Ke}$ Za Zhi 38: 203-206, 2003 (In Chinese).

23. Rödel F, Hoffmann J, Grabenbauer GG, Papadopoulos T, Weiss C, Günther K, Schick C, Sauer R and Rödel C: High survivin expression is associated with reduced apoptosis in rectal cancer and may predict disease-free survival after preoperative radiochemotherapy and surgical resection. Strahlenther Onkol 178: 426-435, 2002.

24. Gradilone A, Gazzaniga P, Ribuffo D, Scarpa S, Cigna E, Vasaturo F, Bottoni U, Innocenzi D, Calvieri S, Scuderi N, et al: Survivin, bcl-2, bax, and bcl-X gene expression in sentinel lymph nodes from melanoma patients. J Clin Oncol 21: 306-312, 2003.

25. O'Connor DS, Schechner JS, Adida C, Mesri M, Rothermel AL, Li F, Nath AK, Pober JS and Altieri DC: Control of apoptosis during angiogenesis by survivin expression in endothelial cells. Am J Pathol 156: 393-398, 2000.

26. Mesri M, Morales-Ruiz M, Ackermann EJ, Bennett CF, Pober JS, Sessa WC and Altieri DC: Suppression of vascular endothelial growth factor-mediated endothelial cell protection by survivin targeting. Am J Pathol 158: 1757-1765, 2001. 